\title{
UNA REFLEXIÓN SOBRE LA FABULACIÓN, EL ARTE Y LA PEDAGOGÍA EN EL COLECTIVO ARTÍSTICO EL CUERPO HABLA
}

\author{
A REFLECTION ON THE MYTH-MAKING FUNCTION, THE ART AND THE \\ PEDAGOGY IN THE ARTISTIC COLLECTIVE EL CUERPO HABLA
}

\author{
UMA REFLEXÃO SOBRE FABULAÇÃO, ARTE E PEDAGOGIA \\ NO COLETIVO ARTÍSTICO O CORPO FALA
}

Diego Alejandro Díez Echavarría ${ }^{1}$

\section{Resumen}

\begin{abstract}
Este es un artículo de reflexión derivado del proyecto de investigación "La fabulación. Acción y conceptualización entre la relación arte y pedagogía”. Aquí nos aproximamos a una relación entre el arte y la pedagogía, desde la performance, en la trayectoria del Colectivo Artístico El Cuerpo Habla. Inicialmente, se presenta una discusión teórica para exponer el problema de investigación que se propone como una conceptualización entre la relación arte y pedagogía. Seguido, hablaremos de las orientaciones teóricas y metodológicas de nuestro trabajo en investigación-creación en artes y, por último, esbozaremos la relación que establecemos entre arte y pedagogía desde la práctica colectiva de la performance como carne, fabulación y acontecimiento. Se dibuja, entonces, en el presente texto, las líneas de un trayecto de investigación. El cual no quiere establecerse como un método a seguir, sino como una mirada a los caminos recorridos en la tarea de investigar con el cuerpo y a través del mismo. Esta es una apuesta por una investigación performática, amplia, que busca construir sus propias estrategias en la elaboración de conocimiento situado y corporal.
\end{abstract}

Palabras clave: fabulación; performance; acontecimiento; carne; comunidad; resistencia

Abstract

This is an article of reflection derived from the project of investigation "The Myth-making Function. Action and conceptualization between the relation art and pedagogy". Here we come closer to the relation between art and pedagogy, from the performance, in the career of the Artistic Collective El Cuerpo Habla. Initially, it presents a theoretical discussion to expose the problem of investigation that is proposed as a conceptualization between the relation art and pedagogy. Followed, we will speak about the theoretical and methodological orientations of our work in investigation-creation in arts and, finally, we will outline the relation that we establish between art and pedagogy from the collective practice of the performance as embodied, myth-making and event. The lines of a research path are then drawn in this text. Which does not want to establish itself as a method to follow, but as a look at the paths traveled in the task of investigating with and through the body. This is a commitment to an extensive performative research, which seeks to build its own strategies in the elaboration of situated and bodily knowledge.

Keywords: myth-making function; performance; event; body; community; resistance

1 Comunicador social de la Universidad de Antioquia e integrante del Colectivo Artístico El Cuerpo Habla. Correo electrónico: diez11diego@ gmail.com. Orcid: https://orcid.org/0000-0002-0544-5039. 


\section{Resumo}

Este é um artigo de reflexão derivado do projeto de pesquisa "Fabulação. Ação e conceitualização entre a relação entre arte e pedagogia". Aqui abordamos uma relação entre arte e pedagogia, a partir da performance, na trajetória do Coletivo Artístico 0 Corpo Fala. Inicialmente, é apresentada uma discussão teórica para expor o problema de pesquisa proposto como uma conceituação entre a relação arte e pedagogia. A seguir, discutiremos as orientações teóricas e metodológicas de nosso trabalho de pesquisa-criação nas artes e, finalmente, delinearemos a relação que estabelecemos entre arte e pedagogia a partir da prática coletiva de performance como carne, fabulação e evento. As linhas de um caminho de pesquisa são então desenhadas neste texto. 0 que não quer se estabelecer como um método a ser seguido, mas como um olhar para os caminhos percorridos na tarefa de investigar com e através do corpo. Esse é um compromisso com uma extensa pesquisa performativa, que busca construir suas próprias estratégias na elaboração do conhecimento situado e corporal.

Palavras-chave: fabulação; performance; evento; carne; comunidade; resistência

Para citar este artículo:

Díez Echavarría, D. A. (2019). Una reflexión sobre la fabulación, el arte y la pedagogía en el Colectivo Artístico El Cuerpo Habla. Lúdica Pedagógica, 30, 31-42. https://doi.org/10.17227/ludica.num30-11102 
INTRODUCCIÓN. ABRIR LÍNEAS

DE FUGA EN LA RELACIÓN ENTRE

\section{EL ARTE Y LA PEDAGOGÍA}

La necesidad de esbozar una relación local entre el arte y la pedagogía es connatural a nuestro hacer, desde el surgimiento de El Cuerpo Habla como taller complementario en la Facultad de Artes, hasta la conformación del colectivo artístico y el semillero de investigación. Nuestra búsqueda entre estos dos temas no ha sido concebida como un proyecto que tiene la intención de establecer un nuevo método de enseñanza-aprendizaje o de investigación-creación, tampoco lo ha sido fijar nuevas verdades. Al contrario, nuestro trabajo ha consistido en valernos de diferentes discursos críticos, exploraciones y ejercicios experimentales, para resistir a las formas hegemónicas del arte y la pedagogía, las cuales ignoran sus repercusiones políticas en las comunidades sociales y académicas.

Nuestra intención es abrir nuevos espacios donde se puedan construir otras formas de ser y hacer, en consonancia con apuestas éticas encarnadas que cuestionen las formas de subordinación epistémicas y materiales de nuestra sociedad. En este sentido, el devenir investigativo de El Cuerpo Habla ha consistido también en tomar distancia de la tradición positivista y estructuralista del saber, y acercarse a epistemologías conectadas con las preocupaciones políticas de quien investiga, orientadas a generar prácticas que permitan la construcción de otras posibilidades de mundo.

En este camino, nos hemos situado de manera móvil, por un lado, en las teorizaciones filosóficas posestructuralistas y los estudios de la performance y, por el otro, entre las discusiones y propuestas de la investigación cualitativa y, recientemente, de la investigación-creación, sin obviar el apoyo conceptual en los diferentes saberes. En este sentido, El Cuerpo Habla no se inscribe en una corriente ni tampoco busca nombrarse con una etiqueta dentro del arte y la academia, en cambio, transita, deviene entre las fuerzas y acontecimientos allí ocurridos para localizarse momentáneamente y luego moverse de acuerdo con las intenciones y deseos que movilizan nuestro hacer como colectivo artístico y académico, pero, sobre todo, como manada y comunidad. Por estas razones, se hace necesario dar cuenta de nuestro trayecto, para dibujar un recorrido, una forma de trabajo, y localizar nuestro punto de vista.

En este camino, hemos elaborado una red conceptual con la cual hemos interpretado e intencionado nuestras apuestas artísticas y pedagógicas como colectivo; así integramos, desde hace dos años aproximadamente, el concepto de fabulación en nuestro trabajo. Con este hemos leído el arte desde su configuración estética y su potencia política, a la vez que se han direccionado los principios éticos del colectivo en su labor pedagógica y artística. A continuación, nos situamos en el concepto de fabulación en relación con la red teórica con la que hemos trabajado.

\section{IMBRICACIONES TEÓRICAS ENTRE LA FABULACIÓN, EL ARTE Y LA PEDAGOGÍA COMO ACONTECIMIENTOS}

Sobre la relación entre el cuerpo, la pedagogía, el arte y la performance se han realizado diferentes reflexiones, algunas de ellas argumentan el uso de la performance comoactivador del cuerpo en la enseñanza (Torres, 2014) y otras proponen una pedagogía performática, al centralizar el arte y el cuerpo en las conceptualizaciones de la dinámica en la educación (Fuset, 2013). Sin embargo, la relación en la que trabaja El Cuerpo Habla se dirige a la exploración del arte y la pedagogía como acontecimientos de fabulación. Esta búsqueda emerge en la realización de una sistematización de la experiencia del colectivo, al preguntarse de qué manera conjugar su hacer artístico, pedagógico y académico para trazar cortes en los campos del saber transitados y mapear su devenir creativo. De este modo, se propone leer con el concepto de fabulación la manera en que se intersecan los diferentes campos de trabajo del colectivo, así como sus apuestas éticas, políticas y estéticas configuradas en su trayectoria.

La fabulación es teorizada por Henry Bergson como una función del hombre que le permite la vida social y la creación de mundos imaginarios, esta se deriva de la inteligencia a la vez que surge como resistencia a la misma. La función fabuladora emerge de la necesidad religiosa del hombre de conectar su experiencia vital con el mundo. En sus palabras:

De hecho, la función fabuladora, que pertenece a la inteligencia y que sin embargo no es inteligen- 
cia pura, tiene precisamente este objeto. Su papel es elaborar la religión de que hemos tratado hasta ahora, la que llamamos estática, y de la que diríamos que es la religión natural, si la expresión no hubiese tomado otro sentido. No tenemos, pues, más que resumir lo dicho para definir esta religión en términos precisos. Es una reacción defensiva de la naturaleza contra lo que podría haber de deprimente para el individuo, y de disolvente para la sociedad, en el ejercicio de la inteligencia (Bergson, 1962, p. 210).

La fabulación, de acuerdo con el autor, es la capacidad del ser humano para vivir en sociedad, es su invención noológica para crear universos de sentido que la inteligencia por sí sola nologra realizar, al correr el riesgo del egoísmo. En este sentido, la razón se desliga de la inteligencia al volverse social y deviene en otra función para preservar la vida:

La especie humana existe merced a que el acto mismo por el cual apareció el hombre, con su inteligencia para fabricar herramientas, con su continuo esfuerzo intelectual, y con el peligro creado por la continuación de este esfuerzo, suscitó asimismo la función fabuladora. (Bergson, 1962, p. 203).

Deleuze retoma la teorización de Bergson para darle una connotación política al concepto de fabulación, en su análisis de las sociedades de control y el poder modular constitutivo de las mismas, él argumenta que la fabulación se hace necesaria en cuanto posibilidad de crear agenciamientos minoritarios; que resistan a la desmembración ontológica causada por la dominación de las mayorías, entendiendo este concepto no en sentido estadístico, sino de manera metonímica, como patrones discursivos y prácticas de dominación hegemónicas que se repiten en una sociedad. Deleuze (2006) invoca a Paul Klee y expresa:

Tal es la función de la fabulación bergsoniana... Sorprender a la gente en fabulación flagrante, captar el movimiento de constitución de un pueblo. Los pueblos no preexisten. En cierto modo, el pueblo es lo que falta, como decía Paul Klee. ¿Existía un pueblo palestino? Según Israel, no. Es obvio que sí existía, pero no es esto lo esencial. Lo esencial es que, desde el momento en que se expulsa a los palestinos de suterritorio, en la medida en que resisten, comienza el proceso de constitución de un pueblo. Esto corresponde exactamente a lo que Perrault llama flagrante delito de fabulación. No hay pueblo que se constituya de otro modo. Esto es lo contrario de las ficcionesestablecidas, que remiten siempre al discurso del colonizador, es el discurso minoritario fabricado con intercesores (p. 107).

Es claro en Deleuze el sentido social de la fabulación al hablar de la falta de un pueblo o, mejor, de múltiples pueblos, del llamado para devenir agenciamientos colectivos en un mundo fragmentado por los embates del poder. La dirección de Deleuze al hablar de la falta de un pueblo no es reformadora, es decir, no busca reintegrar la sociedad en su conjunto, al contrario, pretende ser irruptiva, al conjugarse este concepto con su pensamiento sobre el devenir y la multiplicidad, es decir, sobre la formación de devenires colectivos minoritarios, nodos de resistencia al conjunto de la sociedad que se presenta como mayoría. Como bien argumenta Antonelli (2012):

La meta crítica de la fabulación es la ruptura de la continuidad de los relatos recibidos y las historias hegemónicas, mientras que su función positiva radica en la elaboración de imágenes liberadas de las convenciones narrativas y abiertas a la construcción de nuevos agenciamientos sociales (p. 529).

Devenir colectividades, manadas, sociabilidades es la táctica de resistencia conceptualizada por Deleuze para unir las moléculas de mundos posibles. En esta dirección, son de suma importancia para este pensador los intercesores, esos otros con quienes se fabula y se logran actos de habla colectivos.

Lo esencial son los intercesores. La creación son los intercesores. Sin ellos no hay obra. Pueden ser personas - para un filósofo, artistas o científicos, filósofos o artistas para un científico-, pero también cosas, animales o plantas, como en el caso de Castaneda. Reales o ficticios, animados o inanimados, hay que fabricarse intercesores. Es una serie. Si no podemos formar una serie, aunque sea completamente imaginaria, estamos perdidos. [...] Se precisa algún otro que fabule, a quien se sorprenda en "flagrante delito de fabular". Y entonces se constituye, bien sea entre dos o entre más, un discurso minoritario. (Deleuze, 2006, p. 107).

Esta es precisamente la apuesta que vincula los campos de acción de El Cuerpo Habla: tanto la creación artística como el trabajo académico en investigación y pedagogía, se dirigen a la posibilidad de despertar 
un sentido de colectividad, donde el otro pueda ser un intercesor para fabular múltiples mundos. Así, el arte se convierte en un llamado a la fabulación de un pueblo y la pedagogía toma el rumbo de trabajar la inteligencia para devenir multiplicidades, problematizar la relación de sí mismo y el cuidado del otro como principios éticos y estéticos de vida. Esta es la coyuntura por la que transita el arte y la educación en la actualidad, dar las posibilidades para que los estudiantes construyan estrategias de vida y apuestas éticas en su hacer disciplinar y en su ser social. Tal es la problemática que identifica el profesor Carlos Eduardo Sepúlveda, al analizar la relación entre el arte y la pedagogía en la enseñanza de las artes. Desde su perspectiva, el mundo ya no puede ser pensado desde categorías fijas:

[...] una mirada no-metafísica, no teorética, no científica, debe ser pensado desde la axiología y la estética. El nuevo ámbito no corresponde a una teoría metafísica sino a una ética que defiende lo vivo, que se contextualiza en un mundo complejo y diverso, que se asume no como un imperativo categórico sino como una necesidad vital (Sepúlveda, 2009, p. 15).

Esta coyuntura resuena con la problemática general planteada por Bergson y continuada por Deleuze al hablar de la génesis y la disyunción entre la fabulación y la inteligencia. La primera busca preservar el mundo inaugurado por la evolución de la segunda. Si la inteligencia llevó al individuo a la cúspide de la razón, la fabulación pretende retornarlo a sus cimientos carnales de donde se erigió, para transformar su vida. He aquí el momento en que se produce el acontecimiento y se abre el devenir de la fabulación, al deshacer la inteligencia en multiplicidades. Se genera, entonces, un acto de creación que es el llamado a conformar un pueblo; agenciamientos minoritarios que abren la molaridad de los discursos. Dice Deleuze (1987):

La pequeña separación "presión de la sociedad-resistencia de la inteligencia" definía una variabilidad propia de las sociedades humanas. Ahora bien, sucede que, con ocasión de esta separación, algo de extraordinario se produce o se encarna: la emoción creadora [...] esta se sirve de su juego circular para romper el círculo.

De esta emoción creadora aflora el acontecimiento artístico, el acontecimiento pedagógico como res- puestas de las conexiones de múltiples subjetividades con el cosmos, con la vida y con un rencantamiento del mundo. La fuerza del acto creativo deviene en oleadas de perceptos y afectos que ya no dependen de los autores de la obra, sino de la emoción conjugada que se colectiviza y se materializa en una acción, en un "nuevo ser de sensación" en palabras de (Ordoñez, 2012, p. 137).

\section{INVESTIGACIÓN CUALITATIVA E INVESTIGACIÓN EN ARTES. SITUAR EL CONOCIMIENTO DE MANERA MÓVIL Y TRANSFORMADORA}

El trabajo de investigación que realiza El Cuerpo Habla se sitúa entre los pliegues de la investigación cualitativa y la investigación-creación en artes. Sin fijarse de manera definitiva a un método, se trazan relaciones para armar estrategias metodológicas, cartografías que den cuenta del proceso de investigación.

Pese a la presión, tanto en las ciencias sociales como en las humanidades y las artes, de establecer criterios y métodos científicos de medición, verificación y, en ultimas, de legitimación del conocimiento, la perspectiva de trabajo que asume El Cuerpo Habla se une al cúmulo de experiencias y prácticas de investigación que cuestionan al método científico como único camino válido en el espectro de los saberes. De manera que partimos de perspectivas alternativas en la investigación cualitativa y en la investigación en artes; conocidas por investigar desde otras posiciones epistemológicas que problematizan el modo establecido de producción de conocimiento. Critican las relaciones políticas y de poder ocultas bajo la neutralidad de la ciencia y asumen postulados éticos que parten de un examen de la historia y del deseo de un mundo diferente que resista a las formas de dominación.

Estas formas alternativas no son recientes, en los campos de conocimiento de las ciencias sociales y humanas, empiezan a consolidarse a partir de la segunda mitad del siglo $\mathrm{xx}$, al problematizar, por un lado, el modo de hacer investigación social con las mismas herramientas de las ciencias "duras", apoyadas en un paradigma positivista y, por el otro, al cuestionar el origen colonial de las ciencias sociales y el discurso del progreso tecno-científico promovido por 
el avance de los conocimientos de las ciencias exactas, como exponen Denzin y Lincoln (2012).

Sobre estos cuestionamientos se asentarán otros modos de entender la investigación y de construir conocimiento, siempre en una lucha política con los imperativos institucionales y económicos a los que sirve la academia. Apoyadas en las propuestas filosóficas del interaccionismo simbólico, la hermenéutica, la fenomenología, la semiótica, la deconstrucción, en fin, de las filosofías etiquetadas con el rótulo de postmodernas, las ciencias sociales y las humanidades comenzaron a generar otras perspectivas epistemológicas y otras estrategias y prácticas metodológicas acordes con una visión local, política, ética y estética del conocimiento.

En las artes sucederán los movimientos de vanguardia. Algunos encargados de generar una ruptura con las artes visuales, escénicas y musicales, cuyo resultado fue la mezcla entre ellas y la búsqueda de otros caminos de realización que involucraran al público y rompieran con los supuestos conceptuales de las bellas artes, y su concomitante restricción a la alta cultura y al museo como una forma de experiencia elevada, desconectada de la carne de la sociedad. El happening, el fluxus, el teatro experimental, la pintura corporal y la aparición de manifestaciones performáticas en las artes plásticas y la música suscitaron una atmósfera de revolución en las artes, en cuanto a la separación sujeto-objeto en la realización artística y el compromiso político del artista. Esta convulsión creativa es nombrada por Erika Fischer (2011) como el giro performativo de las artes, en cuyo centro se encuentra un cuestionamiento profundo a las bases del arte, fijadas como una separación entre artista, obra y espectador, para proponer realizaciones escénicas, acontecimientos disruptivos que invitan al ritual y la participación triádica entre artista-obra/acontecimiento-público.

De los diferentes movimientos en los escenarios académicos, artísticos y políticos surgirán corrientes de pensamiento alternativas a las formas instrumentales y positivistas de generación de conocimiento y de creación artística. Entre ellas, se localizan, por una parte, los estudios avanzados de performance, la investigación basada en artes, la investigación-creación, y por la otra, las distintas epistemologías y ontologías emergidas en las ciencias sociales y la filosofía, como en la reivindicación de comunidades étnicas y racializadas por la colonización europea del mundo.

En este nuevo panorama de rupturas, emergencias de saberes y formas de hacer en el arte y la academia, no hay verdades unívocas ni tampoco dogmatismos que pasen desapercibidos; cada movimiento intelectual, artístico y político se mueve entre un campo de fuerzas, por lo que no se pretende una clasificación dentro de una corriente de pensamiento o método. Por lo contrario, se busca generar procesos, trayectos conscientes de las fuerzas con que se interactúa. De manera que el conocimiento como el hacer artístico e investigativo resulte de conexiones parciales, móviles y abiertas, donde el compromiso ético con el mundo se manifiesta en las decisiones metodológicas que construyeron una estrategia, un recorrido de trabajo. Como bien lo argumenta Donna Haraway al hablar del conocimiento situado como estrategia epistemológica de investigación:

No queremos una teoría de poderes inocentes para representar el mundo, en la que el lenguaje y los cuerpos vivan el éxtasis de la simbiosis orgánica. Tampoco queremos teorizar el mundo y, mucho menos, actuar sobre él en términos de Sistema Global, pero necesitamos un circuito universal de conexiones, incluyendo la habilidad parcial de traducir los conocimientos entre comunidades muy diferentes y diferenciadas a través del poder. Necesitamos el poder de las teorías críticas modernas sobre cómo son creados los significados y los cuerpos, no para negar los significados y los cuerpos, sino para vivir en significados y en cuerpos que tengan una oportunidad en el futuro (Haraway, 1995, p. 322).

\section{DESTERRITORIALIZAR² PROYECTOS,} PROVOCAR ACONTECIMIENTOS. LA

\section{ESTRATEGIA DE INVESTIGACIÓN-CREACIÓN ASUMIDA POR EL CUERPO HABLA}

La perspectiva de trabajo del Cuerpo Habla se enmarca en la investigación-creación, un campo de reciente aparición en el discurso académico, con el cual, entre otras cosas, se quiere legitimar el aporte de investigación en artes en los ámbitos institucionales del conocimiento (Santamaría, Chingaté, González,

2 Para introducirse en el concepto de desterritorialización, consultar: http://desterritorialidad.blogspot.com.co/2011/10/hay-quesaber-estar-con-los-pies-en-la.html 
Castellanos, Salazar y Morales, 2011). Sin embargo, este enfoque de investigación, por fortuna, todavía no se encuentra normado por la academia, por lo tanto, se conserva dinámico e incierto (Barriga, 2011), lo cual es una ventaja para conceptualizarlo y potenciarlo estratégicamente.

A diferencia de la investigación basada en artes (arts based research) corriente de investigación en artes originada en Estados Unidos, cuyo fin es utilizar el arte como una herramienta de activismo político (Finley, 2012-2015) o de conjugación de expresiones artísticas en la investigación cualitativa para enriquecer el proceso de generación de conocimiento (Ferreiro, 2013). El Cuerpo Habla asume el arte como un dispositivo y no como una herramienta, así lo expresa la profesora Ángela María Chaverra:

La herramienta la usa el sujeto, tiene un solo uso, por lo tanto, es unívoca. El arte no es una herramienta, va más allá, es un dispositivo. Además, las artes no se utilizan, se viven, por lo tanto, aunque tenga usos metodológicos, no es un método. (Comunicación personal, 14 de abril de 2017).

Aunque el arte en su hibridación con la investigación cualitativa haya derivado como una herramienta con fines cognoscitivos, políticos y expansivos en el hacer de la investigación, desde la perspectiva de El Cuerpo Habla, este es asumido de otra manera. Es cierto que el arte ha provocado rupturas y ha posibilitado la construcción de otras prácticas de investigación cualitativa, como argumentan Hernández (2008) y Ferreiro (2013), sin embargo, para nosotros, el arte tiene otra función en relación con la investigación y el compromiso ético del colectivo. Para El Cuerpo Habla el arte es una vivencia y es un acontecimiento, por más que esté presente en el proceso investigativo, no se reduce a una herramienta, sino que se abre a la multiplicidad como experiencia, al devenir, y no a un fin último. La obra se presenta como un dispositivo abierto, como un detonante que resuena y se expande con la actividad del espectador en una relación de copresencia. Erika Fischer argumenta que el estatuto de la obra de arte se desvanece con el giro performativo de las artes, por lo tanto, ya no se puede hablar de un objeto, sino de una relación entre el artista y los espectadores participantes de una realización escénica. En palabras de la autora:
El giro performativo en las artes difícilmente puede comprenderse con ayuda de las teorías estéticas tradicionales. Aun cuando pueden ser útiles en algunos aspectos, son incapaces de comprender el aspecto crucial de este giro: la transformación de la obra de arte en acontecimiento, y la de las relaciones ligadas a ella: la de sujeto y objeto y la de los estatus material y sígnico (Fischer, 2011, p. 46).

En esta dirección, El Cuerpo Habla tiene mayor resonancia con la investigación-creación y los estudios de performance, en tanto el proceso investigativo se nutre de la capacidad de acontecer, explorar y devenir del arte, en lugar de tratarlo como una herramienta al servicio de un método. Nuestra estrategia de investigación comparte algunas de las construcciones teóricas de los estudios de performance, la investigación-creación y la investigación basada en artes, pero no se reduce a ninguno de los métodos planteados por estas corrientes. Por ejemplo, el cuestionamiento a las estructuras conceptuales dicotómicas características de la ciencia, la inscripción meramente disciplinar para abarcar el estudio o comprensión de un tema, la abstracción del arte y el conocimiento de su contexto social, político y ético, la limitación del lenguaje y, por ende, la capacidad de conocer a lo sígnico, el tratamiento del cuerpo y su corporalidad como un objeto y no como una potencia. Tal como se puede encontrar en Taylor y Fuentes (2011).

La investigación-creación es un campo nuevo en la investigación académica. En Colombia, los investigadores en artes Pedro Pablo Gómez y Edgar Ricardo Lambuley, en una publicación del año 2006, La Investigación en artes y el arte como investigación, problematizan la práctica de investigación-creación. Los autores introducen un grupo de cuestionamientos para pensar la práctica de este tipo de investigación:

¿Cuáles son los componentes ideológicos de los discursos y las prácticas del arte? ¿Por qué a muchos artistas les cuesta tanto trabajo la asociación para la creación colectiva?

¿Es el arte un modo de pensar? ¿Cuál es su estatuto respecto de la ciencia o la filosofía?

¿Por qué el cuerpo es un lugar privilegiado de las estrategias y prácticas artísticas contemporáneas? ¿Dónde radica la importancia de la persona del investigador respecto de su objeto de investiga- 
ción? ¿Por qué se leen tan poco los productos de investigación que produce la comunidad artística? ¿Por qué es tan difícil la socialización de los resultados de la investigación? ¿De qué manera la sociedad puede ser más abierta para el arte? ¿El mundo del arte es un mundo en sí mismo? ¿Por qué la relación entre la producción artística y el pensamiento sobre el arte es tan asimétrica? (Gómez y Lambuley, 2006, p. 12).

Para los investigadores citados, el discurso y la práctica de investigación-creación no se resumen en una generalidad, existen en una multiplicidad y de manera local, quizás por lo novedoso de la temática en las instituciones universitarias y centros de investigación; sin embargo, que las prácticas y los discursos tengan un carácter local y escapen a una síntesis de método y discurso es una oportunidad para no reducir la investigación-creación a una práctica disciplinar cerrada. Construida a merced de los parámetros de la ciencia positiva para ganar legitimidad en las comunidades académicas. A este respecto, El Cuerpo Habla se resiste de manera crítica y, en cambio, apuesta por generar prácticas y estrategias metodológicas, como propone Denzin y Lincoln (2012), tanto en la investigación y en la creación, de acuerdo con nuestras fuentes filosóficas, artísticas y pedagógicas.

El Cuerpo Habla toma la investigación-creación como un proceso emergente durante la apertura y cierre de un problema investigativo, el cual no se agota en encontrar respuestas o entregar soluciones, sino en encarnar un trayecto de experimentación y transformación de quienes participan en él. En esta medida, la creación de una performance se convierte en un proceso reflexivo, y la realización de una investigación se vuelve un proceso vivencial. Ambos maduran en el trabajo de campo, la lectura de textos, la realización de acciones y experimentaciones performáticas, en la convivencia entre el colectivo y la intervención en espacios públicos de ciudad al intervenir en las calles. Esto y otras cosas más que escapan al registro de la experiencia, aquellas sensaciones, afectos y perceptos que inundan la carne en la vivencia de una investigación-creación conforman, en términos generales, la estrategia metodológica de El Cuerpo Habla.

A diferencia de las rutas diseñadas por los programas académicos, que ordenan el proceso de investigación-creación en artes, como muestra Barriga
(2011), nuestra apuesta consiste en generar los elementos de un acontecimiento y disponer al cuerpo para entrar en experienciasde subjetivaciones, tanto personales como colectivas, de modo que se pueda conectar cuerpo y reflexión, hacerlos carne. Así pues, la investigación no se convierte en un proceso que registra y ordena una forma de hacer, la generación de un método, sino que se vuelca al devenir, al caos, al encuentro de nuevos sentidos. La lógica es desterritorializada del centro del saber por otros nodos compuestos de intensidades, incertidumbres, desconocimientos, gustos, incomodidades, afectos, sensaciones; todo aquello que la lógica del pensamiento científico insiste en censurar y descalificar como obstáculos epistemológicos.

Aquí se desplaza la lógica del texto, del logos, para hacer una investigación sin etapas secuenciales, como un cuerpo sin órganos, donde las funciones del cerebro no son solo pensar, ni la de la vista observar, la del tacto sentir, ni la de las manos escribir, registrar. Se puede pensar con el leve dolor de los riñones al permanecer mucho tiempo de pie; con la efusividad de la carne desbordada en un salón oscuro; se puede registrar la experiencia con un gesto, un dibujo, con las palabras suscitadas en la introspección de un taller; se crea a partir del devenir y no de la instrumentalización de un objeto o del cuerpo. La lógica del texto científico queda delineada en la forma en la que los contenidos de los proyectos son presentados, pero no ocupan el lugar central del proceso de investigación. Las múltiples conexiones que pueden realizarse en una experiencia entran a determinar la materialidad de un producto, de un registro, de una sistematización. De este modo, el conocimiento surge como resultado de una transformación y no de una interpretación simple, preestablecida por la lógica de la estructura de la investigación científica. En palabras de Chaverra (2016):

Si los organismos como lo presenta Artaud dependen de una estructura gramática, sometida a una jerarquía unívoca, monoteísta, su propuesta del cuerpo sin órganos creará no solamente una grieta artística, sino también una grieta epistemológica que pondrá en evidencia un pensamiento marcado por el orden, la mathesis y la categorización (p. 165).

Es en el rompimiento con el logos, con el discurso del progreso de la modernidad, que inaugura un 
sujeto que instrumentaliza al cuerpo, al otro y a la naturaleza, donde emerge la propuesta de El Cuerpo Habla, basada en la deconstrucción y el devenir como dimensiones necesariamente exploratorias, experimentales y en permanente construcción, para deshacer y agotar los discursos que constituyen al sujeto, los saberes y al arte.

\section{¿CÓMO APRENDE UNA MANADA? ¿CÓMO VIVENCIA SUS AFECTOS, PERCEPTOS $Y$ SENSACIONES MEDIANTE DISPOSITIVOS DE EXPERIMENTACIÓN Y TRANSFORMACIÓN?}

La relación entre arte y pedagogía, en la cual ha trabajo El Cuerpo Habla durante su existencia, se presenta en la actualidad como un dispositivo que conjuga variedad de elementos teóricos, entre los que se destacan conceptos como re-presentación, encarna-acciones, fabulación, acontecimiento, devenir, resistencia y manada (Chaverra, 2016).

De la ruptura epistémica y metodológica que nombrábamos anteriormente, emerge la orientación de trabajar con dispositivos, en respuesta a buscar una alternativa que se abra al proceso de conocer y hacer en la medida en que se desconoce, se desaprende y se deshace. Esta es la paradoja que media los dispositivos vividos en talleres, entrenamientos y acciones en lugares públicos, donde el cuerpo entra en otra relación con el aprendizaje, el conocimiento de sí y del entorno, en la apertura de grietas para sentirlo fuera de la lógica impuesta por la pedagogía tradicional.

Giorgio Agamben retoma a Foucault y conceptualiza el término dispositivo del siguiente modo:

1. El dispositivo se trata de un conjunto heterogéneo que incluye virtualmente cadacosa, sea discursiva o no: discursos, instituciones, edificios, leyes, medidas policíacas, proposiciones filosóficas. El dispositivo, tomado en sí mismo, es la red que se tiende entre estos elementos.

2. El dispositivo siempre tiene una función estratégica concreta, que siempre está inscrita en una relación de poder.

3. Como tal, el dispositivo resulta del cruzamiento de relaciones de poder y de saber (Agamben, 2011, párrafo 4).
De manera metonímica, El Cuerpo Habla usa la noción de dispositivo en dos sentidos, el primero, para detectar y hackear los entramados de saber-poder que hacen al cuerpo una unidad funcional y moldeable, y, el segundo, para armar nuevas redes a partir de la detonación del cuerpo como unidad-sujeto. Un dispositivo puede manifestarse en una acción, en un taller o unaperformance, pero no se reducen a estas, pues su sentido se encuentra en la multiplicidad de conexiones de las cuales la acción, el taller o la performance hacen parte.

En este sentido, no hay una lógica que represente las interconexiones ni los significados que detona el dispositivo, estas emergen en la interacción entre quienes participan de él. Es poresta razón que el dispositivo, tal como es conceptualizado por El Cuerpo Habla, no es únicamente una red, un entramado; es también un acontecimiento. Sucede sin una determinación directa, sin la prefabricación de un efecto en el otro, más que el generado en su propia experiencia al conjugarse el significado en una tríada de sentido: significante-coyuntura-significado.

Armar los dispositivos con los que trabaja El Cuerpo Habla es un proceso resultado de la praxis, es decir, de la acción, la reflexión y la repetición hermanadas. Un taller puede realizarse muchas veces hasta ajustar las condiciones y las indicaciones necesarias para su realización; en el transcurso puede variar y modificarse hasta lograr un dispositivo. De igual manera, una acción o una performance tienen un proceso de pensamiento, entrenamiento y preparación corporal; este proceso no busca adecuar los cuerpos a una representación, sino disponerlos para entrar, permanecer y salir de un acontecimiento. Una acción o una performance no son escenificaciones teatrales, por lo que escapan a la estructura de un guion, su carácter es de presencia y presentación in situ. Dice Ordoñez (2011) que: "El acontecimiento desborda siempre los límites del esquema comprensivo preexistente mediante el cual se trata de aferrarlo, de especificarlo, de modo que una y otra vez es preciso ajustar los procedimientos de explicación y expresión" (p. 133).

Estos dispositivos tienen una connotación política y ética, pues El Cuerpo Habla entiende que no es posible cuestionar al sujeto y las prácticas de subjetivación engendradas en la modernidad y manifiestas en 
las instituciones que nos gobiernan, sin que el sujeto mismo se transforme y devenga en subjetivaciones que le permitan resistir a la barbarie civilizatoria y al control modular de las instituciones políticas y económicas del siglo XXI, que precarizan la vida planetaria (Deleuze, 2006). Como explica Chaverra:

En consecuencia, la recurrencia a autores que abren la mirada de lo humano más alláde una caracterización fija y lo relacionan con el cosmos, la tierra, la pertenencia al mundo, ayuda a entender una visión más holística. El devenir, el fabular, el autoinventar declara al sujeto en pertenencia con un contexto, una comunidad, con la tierra, lo invita a ser manada. [...] Más allá del yo, se genera una pregunta por la capacidad de devenir animal, agua, tierra, otro y en ese instante en que se da la acción fabuladora, el acontecimiento -en este caso artístico-, apunta a una anulación psicológica de una identidad fija, del antropomorfismo, para entrar en otros estados. (2016, p. 148).

Es aquí donde hemos conjugado últimamente el concepto de fabulación como una posibilidad en el discurso de orientar los procesos investigativos hacia una transformación social, al igual que potenciar la creación artística desde el llamado a un pueblo, a un acontecimiento donde lavisión de otros mundos sea posible, argumenta la profesora Ángela María Chaverra:

Gregg Lambert, lector de Deleuze, sostiene, en este sentido, que la fabulación como se puede interpretar en la contemporaneidad nunca es una cuestión de escapar del mundo que existe, ni de la verdad que lo sostiene, ni la creación de una verdad superior, sino abonar el campo para la expresión de otros mundos posibles, los cuales generen nuevas maneras de pensar y alteren la forma como se construye la realidad, para realizar variaciones al mundo dado por verdadero (2016, p. 145).

En la contemporaneidad se cuestiona la función del arte, y El Cuerpo Habla ha respondido a este cuestionamiento desde la perspectiva de autores que problematizan las implicaciones políticasy éticas del arte desde la fabulación. Bergson, Nietzsche, Benjamin, Ranciere y Deleuze despojan el arte del egoísmo, de la inteligencia personal, de la vanidad que da la razón al sujeto, para transformarlo en una propuesta social, una resistencia que permita la pér- dida del aura, para ellos, el arte se presenta como ritual y proceso de transformación (2016, p. 143).

Encarnar el mundo en acontecimientos de fabulación:

La educación es el punto en el que decidimos si amamos el mundo lo bastante como para asumir una responsabilidad por él y así salvarlo de la ruina que, de no ser por la renovación, de no ser por la llegada de los nuevos y los jóvenes, sería inevitable. También por la educación decidimos si amamos a nuestros hijos lo bastante como para no arrojarlos de nuestro mundo y librarlos a sus propios recursos, ni quitarles de las manos la oportunidad de emprender algo nuevo, algo que nosotros no imaginamos, lo bastante como para prepararlos con tiempo para la tarea de renovar un mundo común. (Arendt, 2003, p. 208).

Como expresa Hannah Arendt, en la educación emerge la capacidad de amar al mundo y asumir una responsabilidad en él y con él. Parte de este postulado se retoma en el accionar de El Cuerpo Habla, hacer un llamado, parafraseando a Deleuze (2006, p. 147), a un nuevo régimen de signos, un régimen fabuloso que busque trabajar contra los regímenes hegemónicos instituidos. A partir de la fabulación como acontecimiento artístico y vital se quiere invitar a la formación de una colectividad, a la detonación de la unidad del cuerpo para estallar en la propia carne y hacer parte de la carne del mundo. Las instituciones educativas, como parte de los regímenes disciplinares inaugurados en la Modernidad, han atrofiado la experiencia del cuerpo al convertirlo en una unidad igual a un yo, al tiempo que lo volvía una materia dócil tanto para el trabajo como para el consumo. Es por esto, que se hace necesaria una pedagogía, no solo del acontecimiento y de la fabulación, sino de la incomodidad. Dice Barba (1999):

En el terreno de la comodidad no hay forma de crear ni de conseguir una presencia total. Al encontrar el punto de comodidad el cuerpo inmediatamente suelta sus canales de energía y las zonas de las que emerge la energía como las articulaciones, el centro (las caderas), la flexión de las rodillas, la oposición entre la coronilla y las plantas de los pies. Dentro del entrenamiento el actor está en constante riesgo y una tarea básica es la búsqueda de la incomodidad. Es el momento de la pérdida del equilibrio, donde resuelve una postura extra-cotidiana, donde vive el músculo que no tenemos consciente o que posible- 
mente no utilizamos a diario. Es la situación límite del cuerpo donde la energía se expande (p. 18).

De acuerdo con Barba, buscar la activación del cuerpo, sacarlo del lugar fijo de su condición disciplinada, es una parte complementaria de la actividad pedagógica de El Cuerpo Habla, en cuanto se busca romper con los condicionamientos que impiden el fabular, el devenir y el hacerse responsable del mundo. Pero, para ello, es necesario movilizar la carne, potenciar el fluir como un trayecto donde se deshacen los regímenes de subjetividad para devenir un mundo fabulado desde el deseo personal y colectivo de otra realidad. Parafraseando a Ángela Chaverra (2016, p. 191), la educación tradicional se ha enfocado en la capacidad de adaptación de las personas a partir de unas competencias. Desde este modelo, se universaliza una verdad y se despliega una manera de enseñar y de aprender, legitimada por el Estado. Una dislocación de este modelo es generar una pregunta sobre sí, que lleve a una inquietud del ser y, por lo tanto, a un cuidado de sí y del otro. Una pedagogía del cuidado y de la alteridad.

El cuidado de sí y del otro es un componente fundamental de la apuesta pedagógica y artística de El Cuerpo Habla, no sería coherente invitar a la fabulación de otras posibilidades de vida, sin tener en cuenta las implicaciones éticas de cuidarse a sí mismo y al otro. No se puede construir colectividad si el otro no es respetado, valorado e interpelado al esperar de él la corresponsabilidad necesaria para convivir, tanto en los momentos de celebración como de conflicto y controversia. Desacomodar, cuestionar, deshacer la propia identidad no es un camino fácil, su trayecto se aboca a la incertidumbre, por lo que es necesario el apoyo y la potencia que se genera en la colectividad, en la manada, para devenir junto con el otro, cada uno en su proceso, sin afanes ni obligaciones más que el compromiso asumido de fabular el mundo, en la búsqueda de otras maneras encarnadas de habitarlo y transformarlo.

\section{REFERENCIAS}

Agamben, G. (2011). ¿Qué es un dispositivo? Sociológica, 26(73), 249-264. http://www.scielo.org.mx/pdf/soc/ v26n73/v26n73a10.pdf
Antonelli, M. (2012). El problema de la utopía en Gilles Deleuze. Isegoría. Revista de Filosofía Moral y Política, 47, 519-539.

Arendt, H. (2003). La condición humana. Buenos Aires: Paidós.

Barba, E. (1999). La canoa de papel: tratado de antropología teatral. Buenos Aires: Ediciones Catálogos.

Barriga, M. (2011). La investigación creación en los trabajos de pregrado y postgrado en educación artística. $E l$ Artista, 8, 317-330. http://www.redalyc.org/articulo. oa?id=87420931021

Bergson, H. (1962). Las dos fuentes de la moral y la religión. Buenos Aires: Editorial Sudamericana.

Chaverra, A. (2016). El cuerpo habla. [Tesis de doctorado, Universidad de Antioquia].

Deleuze, G. (1987). El bersgsonismo. Madrid: Ediciones Cátedra.

Deleuze, G. (2006). Conversaciones 1972-1990. España: Pretextos.

Denzin, N. y Lincoln, Y. (2012). Introducción general. La investigación cualitativa como disciplina y como práctica. En Manual de investigación cualitativa (vol. I, pp. 43-101). España: Gedisa.

Hernández, F. (2008). La investigación basada en las artes. Propuestas para repensar la investigación en educación. Educatio Siglo v, 26, 85-118.

Ferreiro, A. (2013). Una mirada a la investigación cualitativa desde las artes. Algunos aportes metodológicos y conceptuales de las artes escénicas. Societarts. Revista de Artes y Humanidades, 2(4), 3-24.

Finley, S. (2015). Investigación con base en las artes. En N. Denzin y Y. Lincoln (coords.), Manual de investigación cualitativa (vol. IV. Métodos de recolección y análisis de datos). España: Gedisa.

Fischer, E. (2011). Estética de lo performativo. Madrid: Abada Editores.

Fuset, M. (2013). Pedagogía performática y patrimonio artístico. Educación Artística: Revista de Investigación (EARI), 4, 89-98.

Gómez, P. y Lambuley, E. (2006). La investigación en artes y el arte como investigación. Bogotá: Universidad Distrital Francisco José de Caldas.

Haraway, D. (1995). Conocimientos situados: la cuestión científica en el feminismo y el privilegio de la perspectiva parcial. En Ciencia, cyborgs y mujeres. La reinven- 
ción de la naturaleza (trad. M. Talens, pp. 313-346). Madrid: Cátedra.

Ordoñez, L. (2012). Arte y acontecimiento. Una aproximación a la estética deleuziana. Revista Latinoamericana de Filosofía, 37(1), 127-152.

Santamaría, D., Chingaté, D., González, J., Castellanos, N., Salazar, M. y Morales, S. (2011). La productividad de las artes en las universidades colombianas: desafíos a los mecanismos de medición del conocimiento. Cuadernos de Música, Artes Visuales y Artes Escénicas, 6(2), 87- 116 .
Sepúlveda, C. (2009). Experiencia como campo de relación entre el arte y la pedagogía. (Pensamiento), (Palabra) y Obra, 1(1), 12-18.

Taylor, D. y Fuentes, M. (2011). Introducción. En Estudios avanzados de performance (pp. 7-30). México: Fondo de Cultura Económica.

Torres, C. (2014). La práctica artística como dispositivo de acción política. AusArt Journal for Research in Art, $2(2), 22-34$. 